\title{
Phase II monitoring of multivariate simple linear profiles with estimated parameters
}

\author{
Ahmad Ahmadi Yazdi ${ }^{1}$. Ali Zeinal Hamadani ${ }^{1} \cdot$ Amirhossein Amiri $^{2}$
}

Received: 29 May 2018 / Accepted: 17 February 2019 / Published online: 26 February 2019

(c) The Author(s) 2019

\begin{abstract}
In some applications of statistical process monitoring, a quality characteristic can be characterized by linear regression relationships between several response variables and one explanatory variable, which is referred to as a "multivariate simple linear profile." It is usually assumed that the process parameters are known in Phase II. However, in most applications, this assumption is violated; the parameters are unknown and should be estimated based on historical data sets in Phase I. This study aims to compare the effect of parameter estimation on the performance of three Phase II approaches for monitoring multivariate simple linear profiles, designated as MEWMA, MEWMA_3 and MEWMA $/ \chi^{2}$. Three metrics are used to accomplish this objective: AARL, SDARL and CVARL. The superior method may be different in terms of the AARL and $S D A R L$ metrics. Using the CVARL metric helps practitioners make reliable decisions. The comparisons are carried out under both in-control and out-of-control conditions for all competing approaches. The corrected limits are also obtained by a Monte Carlo simulation in order to decrease the required number of Phase I samples for parameter estimation. The results reveal that parameter estimation strongly affects the in-control and out-of-control performance of monitoring approaches, and a large number of Phase I samples are needed to achieve a parameter estimation that is close to the known parameters. The simulation results show that the MEWMA and MEWMA $/ \chi^{2}$ methods perform better than the MEWMA_3 method in terms of the $C V A R L$ metric. However, the superior approach is different in terms of $A A R L$ and $S D A R L$.
\end{abstract}

Keywords Profile monitoring $\cdot$ Multivariate simple linear profiles $\cdot$ Estimation effect $\cdot$ Average run length $\cdot$ Statistical process monitoring $\cdot$ Phase II analysis

\section{Introduction}

In most statistical process monitoring (SPM) applications, it is assumed that the quality of a process or product is characterized by the statistical distribution of a single quality characteristic or a vector of several quality characteristics. However, in some cases, process quality could be characterized by a functional relationship between a response variable and one or more explanatory variables, which is referred to as a "profile." Similar to other process monitoring approaches, profile monitoring is carried out by sampling. Several sets of data points are collected to represent their relationship with

Amirhossein Amiri

amiri@shahed.ac.ir

1 Department of Industrial and Systems Engineering, Isfahan University of Technology, Isfahan 8415683111, Iran

2 Department of Industrial Engineering, Faculty of Engineering, Shahed University, Tehran, Iran a curve (profile). The main objective of profile monitoring is to monitor the stability of this curve over time.

There are different types of profiles. If a linear regression model can represent the relationship between one response variable and one explanatory variable that is a simple linear profile. As an application of simple linear profiles, Kang and Albin (2000) addressed a calibration problem in semiconductor manufacturing and monitored the relationship between measured pressure $(Y)$ and the amount of flow $(X)$ using profiles. Monitoring and change point estimation of simple linear profiles have been considered by some researchers, including Stover and Brill (1998), Mestek et al. (1994), Mahmoud and Woodall (2004), Wang and Tsung (2005), Gupta et al. (2006), Mahmoud et al. (2007), Zou et al. (2007), Noorossana et al. (2008), Jensen et al. (2008), Narvand et al. (2013), Khedmati and Niaki $(2015,2016)$ and Kalaei et al. (2018).

There are other more complicated types of profiles such as multiple, polynomial and nonlinear profiles that have 
been studied by many researchers. Several approaches to monitor multiple linear profiles have been developed by some researchers, including Mahmoud (2008), Jensen et al. (2008), Zou et al. (2007), Parker and Finley (2007) and Amiri et al. (2012). In addition, Kazemzadeh et al. (2008, 2009) proposed several methods for monitoring polynomial profiles. Nonlinear profile monitoring through parametric and nonparametric methods and mixed linear profiles has been studied by some researchers, including Ding et al. (2006), Jeong et al. (2006), Williams et al. (2007), Moguerza et al. (2007) and Vaghefi et al. (2009).

In the case of multivariate simple linear (MSL) profiles, which are the main focus of the current paper, the term "simple" refers to the uniqueness of the explanatory variable, and the term "multivariate" refers to the multiplicity of the response variable. In such profiles, there are several response variables, each of which has a linear regression relationship with one explanatory variable. Some researchers, such as Noorossana et al. (2010), Zou et al. (2012), Ayoubi et al. (2014) and Adibi et al. (2014), have studied different aspects of multivariate profile monitoring.

Noorossana et al. (2010) were the first researchers who proposed three methods, designated as MEWMA_3, MEWMA $/ \chi^{2}$ and MEWMA, for monitoring MSL profiles in Phase II. They evaluated the performance of these methods in detecting shifts in profile parameters under in-control and out-of-control conditions in terms of average run length $(A R L)$. Their results showed that the MEWMA and MEWMA $/ \chi^{2}$ methods outperform MEWMA_3. They also used these three methods in a real case study.

Based on the literature, profile monitoring approaches are divided into two phases: Phase I and Phase II. These phases are distinguished by the difference in their goals. In Phase I, a set of historical data points is available ( $m$ samples, each based on $n$ observations). The main goals of Phase I are evaluating process stability, recognizing and eliminating assignable causes and estimating process parameters from in-control samples. The objective of Phase I is to recognize assignable causes with high probability. The main interest in Phase II is quick detection of shifts based on the estimated parameters obtained in Phase I. Existing monitoring approaches in these two phases are dissimilar and use different evaluation metrics. In Phase I, the probability of a signal under out-of-control conditions is used to describe the ability of a control chart to detect shifts in parameters. The ARL metric and its statistical properties are applied to evaluate control chart performance in Phase II. To find out more about profile monitoring approaches in Phases I and II, see the review paper by Woodall et al. (2004) and Woodall 2007) and the book edited by Noorossana et al. (2011).

In most studies of Phase II profile monitoring, it is assumed that the in-control parameters are known. In fact, the values of profile parameters are rarely known in practical environments and should be estimated in Phase I. This assumption may affect control chart performance because of the additional variability added by estimation of parameters. Therefore, the following questions should be answered:

- Does profile parameter estimation really affect the performance of Phase II control chart schemes?

- What is the proper metric for measuring the effect of parameter estimation?

- Which method of profile monitoring is less affected by parameter estimation?

There are many studies in which the effect of parameter estimation on the performance of control charts is estimated for quality characteristics except profiles, including Burroughs et al. (1993), Chen (1997), Chakraborti (2000), Shishebori and Zeinal Hamadani (2009), Shishebori et al. (2015), Jones et al. (2001, 2004), Shu et al. (2004), Jones (2002), Zhang and Chen (2002), Zwetsloot and Woodall (2017), Khoo (2005), Castagliola et al. (2016), Saleh et al. (2015). For information on more research on this area, see the following review papers: Jensen et al. (2006) and Psarakis et al. (2014).

While a substantial number of studies have been done on evaluating the effect of parameter estimation on the performance of control charts for non-profile characteristics, there are only a few studies on this topic for profile characteristics. Woodall and Montgomery (2014) discussed the field of statistical process control, stating that "There is also work needed on the effect of parameter estimation error on the Phase II performance of profile monitoring methods."

Mahmoud (2012) was the first author to compare the incontrol and out-of-control performance of three well-known simple linear profile monitoring approaches that had first been proposed by Kang and Albin (2000), Kim et al. (2003) and Mahmoud et al. (2010), for use when parameters are estimated. In this study, the $A R L$ and standard deviation of run length (SDRL) metrics are used to compare the performance of simple linear profile monitoring approaches. The results showed that using estimated profile parameters instead of known values in Phase II strongly affects the performance of all three methods under in-control and outof-control conditions in terms of both the $A R L$ and $S D R L$ metrics. This author also used simulated corrected limits to investigate the out-of-control performance of the monitoring approaches. Corrected limits are usually wider than control limits based on known parameters and reflect variability added to the process by parameters estimation. A smaller number of Phase I samples are needed for proper estimation when the corrected limits are applied. The simulation results showed that the method described by Mahmoud et al. (2010) has better out-of-control run length performance than other competing methods. 
Mahmoud (2012) used only the $A R L$ and SDRL metrics to compare control chart performance based on estimated parameters. However, the standard deviation of average run length (SDARL) is also important in comparison with control chart performance. When different practitioners take samples in Phase I, they may estimate different values for process parameters. Consequently, they may obtain different values for in-control $A R L$, adding a new source of variability to the process: practitioner-to-practitioner variability. In other words, when it is assumed that the process parameters are unknown in Phase II and should be estimated from the Phase I data set, the $A R L$ is no longer a parameter, but becomes a random variable. The $A R L$ curve has almost a right-skewed distribution (Jensen et al. 2006). Zhang et al. (2014) maintained that practitioner-to-practitioner variation is inevitable because different Phase I data sets are used. The $S D A R L$ is a very useful metric for measuring this variation. Researchers have suggested that the SDARL should be within $5-10 \%$ of the desired in-control $A R L$ value. It is obvious that when the process parameters are known, the SDARL is equal to zero. Aly et al. (2015), similar to Mahmoud (2012), compared the performance of three methods of simple linear profile monitoring [the method proposed by Kang and Albin (2000), Kim et al. (2003) and Mahmoud et al. (2010)] in terms of in-control AARL and SDARL, but they did not investigate the out-of-control performance of the mentioned control charts. The results of their study showed that when the parameters are estimated, the Kim et al. (2003) method generally shows better in-control performance compared to the other competing methods in terms of the SDARL. They also illustrated that if a control chart shows a particular performance in terms of the $A R L$, it may not show the same performance in terms of the $S D A R L$.

The coefficient of the variation of average run length (CVARL), along with the AARL and SDARL, has also been used to evaluate the performance of multivariate adaptive EWMA control charts by Aly et al. (2016). The CVARL is calculated by the following equation:

$C V A R L=\frac{S D A R L}{A A R L} \times 100$.

To the best of the authors' knowledge, there is no study in the literature that evaluates the effect of parameter estimation on the performance of control charts for monitoring MSL profiles. This paper can be an useful source for quality control engineers in choosing the best control chart for monitoring MSL profiles. According to the simulation results, using parameter estimates with upper control limits designed based on the known parameters can result in a significant deterioration of the chart performance. Because these estimators add extra variability in the chart control limit(s). Therefore, applying a control chart scheme which is less affected by this effect would be very constructive and leads to a huge cost saving in terms of time or expenses for any manufacturing systems. Measuring the estimation effect can be carried out by computing some metrics. In this paper, for the first time, the $A A R L$, $S D A R L$ and $C V A R L$ are used to evaluate the in-control and out-of-control performance of control chart schemes. Note that, using different metrics such as the $A A R L$ or $S D A R L$ may lead to different results, which can lead to ambiguity in choosing the best method. In this situation, using the CVARL, which considers both the AARL and SDARL metrics, can help achieve a reliable decision and choose the best method.

According to the literature, it is obvious that the large Phase I sample leads to more accurate estimates and, consequently, better Phase II performance. However, there might be situations in which collecting a large number of samples is not possible. Hence, using corrected control limits is suggested; in this procedure, a large number of $m$ Phase I data are not required to achieve the desired incontrol $A R L$. Using wider control chart limits to reflect the variability of parameters estimation is the main idea of using corrected limits. In previous studies such as Mahmoud (2012), corrected limits have been obtained in order to achieve an in-control value of $A R L=200$. However, in the current study, corrected limits are established in order to achieve an in-control value of $A A R L=200$ through simulation runs to consider practitioner-to-practitioner variability and reduce the number of Phase I samples for parameters estimation. Note that the out-of-control performance of monitoring approaches is also evaluated based on simulated corrected limits.

The rest of this paper is organized as follows. "The multivariate simple linear regression model" section contains a brief explanation of the multivariate simple linear regression model. In "Phase II monitoring methods for multivariate simple linear profiles" section, three MSL profile monitoring approaches in Phase II are presented. "The proposed approach for measuring the effect of parameter estimation on the performance of Phase II control charts" section presents a description of the procedure for evaluating the effect of parameter estimation on the performance of monitoring approaches. Then, "Comparison of control chart performance under in-control conditions" section presents the in-control performance of competing approaches in terms of the AARL, SDARL and CVARL metrics. "The proposed approach for establishing corrected limits" section presents the simulated corrected limits to achieve the desired in-control AARL. In "Comparison of control chart performance under out-of-control conditions" section, the detection performance of the control chart schemes is compared under different types of shifts. Finally, the conclusions and suggestion for further research are provided in "Conclusion and suggestions for future research" section. 


\section{The multivariate simple linear regression model}

In this model, there is a linear regression relationship among several response variables and one explanatory variable in an MSL profile. Assume that $m$ Phase I samples are available. In each sample, there are $n$ fixed values for explanatory variable $(x)$, and for each value of $n$, there are $p$ corresponding values of the response variables. Therefore, for the $k$ th sample, $n$ observations are available as follows:

$\left(x_{i}, y_{i 1 k}, y_{i 2 k}, \ldots, y_{i p k}\right), \quad i=1,2, \ldots, n \quad k=1,2, \ldots$,

where $x_{i}$ is $i$ th value of the explanatory variable and $y_{i 1 k}, y_{i 2 k}, \ldots, y_{i p k}$ are corresponding values of the response variables. The relationship among the explanatory and response variables can be represented as:

$\mathbf{Y}_{k}=\mathbf{X B}+\mathbf{E}_{k}, \quad k=1,2, \ldots$,

or

$$
\begin{aligned}
{\left[\begin{array}{cccc}
y_{11 k} & y_{12 k} & \ldots & y_{1 p k} \\
y_{21 k} & y_{22 k} & \ldots & y_{2 p k} \\
\vdots & \vdots & \ddots & \vdots \\
y_{n 1 k} & y_{n 2 k} & \ldots & y_{n p k}
\end{array}\right]=} & {\left[\begin{array}{cc}
1 & x_{1} \\
1 & x_{2} \\
\vdots & \vdots \\
1 & x_{n}
\end{array}\right]\left[\begin{array}{cccc}
\beta_{01} & \beta_{02} & \cdots & \beta_{0 p} \\
\beta_{11} & \beta_{12} & \cdots & \beta_{1 p}
\end{array}\right] } \\
& +\left[\begin{array}{cccc}
\varepsilon_{11 k} & \varepsilon_{12 k} & \ldots & \varepsilon_{1 p k} \\
\varepsilon_{21 k} & \varepsilon_{22 k} & \ldots & \varepsilon_{2 p k} \\
\vdots & \vdots & \ddots & \vdots \\
\varepsilon_{n 1 k} & \varepsilon_{n 2 k} & \cdots & \varepsilon_{n p k}
\end{array}\right]
\end{aligned}
$$

where $\mathbf{Y}_{k}=\left(\boldsymbol{y}_{1 k}, \boldsymbol{y}_{2 k}, \ldots, \boldsymbol{y}_{n k}\right)^{\mathrm{T}}$ is an $n \times p$ matrix of the response variables for the $k$ th sample. Each row of $\mathbf{Y}_{k}\left(\boldsymbol{y}_{i k}\right)$ is related to one of the $x_{i}$ values, which contains $p$ values of the response variables. In addition, $\mathbf{X}=\left[\begin{array}{ll}\boldsymbol{x}\end{array}\right]$ is an $n \times 2$ matrix of the explanatory variables in which $\boldsymbol{x}$ is a vector of $x$ values. It is assumed that $\mathbf{X}=\left[\begin{array}{ll}1 & \boldsymbol{x}\end{array}\right]$ is fixed in all sampling points. $\mathbf{E}_{k}=\left(\varepsilon_{1 k}, \varepsilon_{2 k}, \ldots, \varepsilon_{n k}\right)^{\mathrm{T}}$ is an $n \times p$ matrix of error terms for the $k$ th sample in which each row $\left(\varepsilon_{i k}\right)$ corresponds to one row of the response variable matrix $\left(\mathbf{Y}_{k}\right)$. Based on the assumptions of the multivariate regression model, each $\varepsilon_{i k}$ follows a multivariate normal distribution with a $1 \times p$ mean vector of zero and a $p \times p$ covariance matrix $\boldsymbol{\Sigma}$. In fact, the elements of each row of $\mathbf{E}_{k}$ are dependent on each other by a multivariate normal distribution; however, each row is independent of the other rows. $\mathbf{B}_{k}=\left(\boldsymbol{\beta}_{0 k}, \boldsymbol{\beta}_{1 k}\right)^{\mathrm{T}}$ is a $2 \times p$ matrix of known simple regression coefficients. Based on the least squares approach, the estimation of $\mathbf{B}_{k}$ can be calculated by the following relation:

$$
\begin{aligned}
\hat{\mathbf{B}}_{k} & =\left(\mathbf{X}^{\mathrm{T}} \mathbf{X}\right)^{-1} \mathbf{X}^{\mathrm{T}} \mathbf{Y}_{\mathrm{k}}=\left(\hat{\boldsymbol{\beta}}_{0 k}, \hat{\boldsymbol{\beta}}_{1 k}\right)^{\mathrm{T}}=\left[\begin{array}{lll}
\hat{\beta}_{01 k} & \cdots & \hat{\beta}_{0 p k} \\
\hat{\boldsymbol{\beta}}_{11 k} & \cdots & \hat{\beta}_{1 p k}
\end{array}\right] \\
& =\left[\begin{array}{lll}
\bar{y}_{.1 k}-\hat{\boldsymbol{\beta}}_{11 k} \bar{x} & \cdots & \bar{y}_{. p k}-\hat{\beta}_{1 p k} \bar{x} \\
\frac{S_{x y(1)}}{S_{x x}} & \cdots & \frac{S_{x y(p)}}{S_{x x}}
\end{array}\right],
\end{aligned}
$$

where $\bar{y}_{. j k}=n^{-1} \sum_{i=1}^{n} y_{i j k}, S_{x y(j)}=\sum_{i=1}^{n}\left(x_{i}-\bar{x}\right) y_{i j k}, \quad j=1$, $2, \ldots, p$, and $S_{x x}=\sum_{i=1}^{n}\left(x_{i}-\bar{x}\right)^{2}$.

\section{Phase II monitoring methods for multivariate simple linear profiles}

Noorossana et al. (2010) proposed three control chart approaches for monitoring MSL profiles in Phase II. Then, they evaluated the performance of the proposed methods in terms of the $A R L$ metric under in-control and out-of-control conditions. These three approaches are briefly discussed below.

\section{MEWMA control chart}

This method was first introduced by Lowry et al. (1992). B can be rewritten by a $1 \times 2 p$ vector, namely $\boldsymbol{\beta}_{k}^{\mathrm{T}}$, as follows:

$\boldsymbol{\beta}_{k}^{\mathrm{T}}=\left(\beta_{01 k}, \beta_{02 k}, \ldots, \beta_{0 p k}, \beta_{11 k}, \beta_{12 k}, \ldots, \beta_{1 p k}\right)$.

The least squares error estimate of $\boldsymbol{\beta}_{k}^{\mathrm{T}}$ is $\hat{\boldsymbol{\beta}}_{k}^{\mathrm{T}}$, where it follows a multivariate normal distribution with mean vector of $\boldsymbol{\beta}^{\mathrm{T}}=\left(\beta_{01}, \beta_{02}, \ldots, \beta_{0 p}, \beta_{11}, \beta_{12}, \ldots, \beta_{1 p}\right)$ and $2 p \times 2 p$ covariance matrix $\boldsymbol{\Sigma}_{\hat{\beta}}$. The formulas for computing the elements of $\boldsymbol{\Sigma}_{\hat{\boldsymbol{\beta}}}$ were given in their paper. Finally, the $\boldsymbol{z}_{k}$ statistic is given as follows (Noorossana et al. 2010):

$\boldsymbol{z}_{k}=\theta\left(\hat{\boldsymbol{\beta}}_{k}-\boldsymbol{\beta}\right)^{\mathrm{T}}+(1-\theta) \boldsymbol{z}_{k-1}, \quad k=1,2, \ldots$,

where $z_{k}$ is a $1 \times 2 p$ matrix and $\theta(0<\theta \leq 1)$ is a smoothing parameter. It can be shown that under in-control conditions, $\boldsymbol{z}_{k}$ follows a $2 p$ multivariate normal distribution with a mean vector of zero and a covariance matrix $\boldsymbol{\Sigma}_{z_{k}}=\left\{\frac{\theta}{(2-\theta)}\right\} \boldsymbol{\Sigma}_{\hat{\boldsymbol{\beta}}}$. Hence, for $k$ th sample, the MEWMA statistic is obtained by using the following equation (Noorossana et al. 2010):

$T_{z_{k}}^{2}=z_{k} \Sigma_{z_{k}}^{-1} z_{k}^{T}, \quad k=1,2, \ldots$

In this method, the chart alarms when $T_{z_{k}}^{2}>h_{\beta}$. The value of $h_{\beta}$. is determined through the simulation to achieve a specified in-control $A R L$.

\section{MEWMA $/ \chi^{2}$ control chart}

This method is an extension of the second approach proposed by Kang and Albin (2000). The vector of the average error for the $k$ th sample is denoted by $\overline{\boldsymbol{e}}_{k}=\left(\bar{e}_{1 k}, \bar{e}_{2 k}, \ldots, \bar{e}_{p k}\right)^{\mathrm{T}}$, in which $\bar{e}_{j k}=\frac{1}{n} \sum_{i=1}^{n} e_{i j k}$. It can be shown that under in-control conditions, $\overline{\boldsymbol{e}}_{k}$ follows a $p$-variate normal distribution with a mean vector of zero and a covariance matrix $\boldsymbol{\Sigma}_{\bar{e}}=n^{-1} \boldsymbol{\Sigma}$.

The MEWMA method proposed by Lowry et al. (1992) is used to monitor average error. For the $k$ th sample, the $1 \times p$ 
vector of weighted moving average errors $\left(z_{k, e}\right)$ is given by (Noorossana et al. 2010):

$z_{k, e}=\theta \overline{\boldsymbol{e}}_{k}+(1-\theta) z_{k-1, e}, \quad k=1,2, \ldots$,

where $z_{0, e}$ is a $1 \times p$ vector and $\theta(0<\theta \leq 1)$ is a smoothing parameter. It can be shown that under in-control conditions, $z_{k, e}$ follows a $p$-variate normal distribution with a mean vector of zero and a covariance matrix $\boldsymbol{\Sigma}_{z, e}=\left\{\frac{\theta}{(2-\theta)}\right\} \boldsymbol{\Sigma}_{\bar{e}}=\left\{\frac{\theta}{n(2-\theta)}\right\} \boldsymbol{\Sigma}$. For the $k$ th sample, the MEWMA statistic is obtained by (Noorossana et al. 2010):

$T_{z_{k, e}}^{2}=z_{k, e} \boldsymbol{\Sigma}_{z_{k, e}}^{-1} z_{k, e}^{T}, k=1,2, \ldots$

The chart signals as soon as $T_{z_{k, e}}^{2}>h_{e}$. The value of $h_{e}$ is determined through the simulation to achieve a specified in-control $A R L$.

Noorossana et al. (2010) developed the method proposed by Noorossana et al. (2004) for monitoring process variability and proposed a new Chi-square statistic, $\chi_{i k}^{2}=\boldsymbol{e}_{i k} \boldsymbol{\Sigma}^{-1} \boldsymbol{e}_{i k}^{\mathrm{T}}$, which follows a Chi-square distribution with $p$ degrees of freedom; consequently, $\chi_{k}^{2}=\sum_{i=1}^{n} \chi_{i k}^{2}$ follows a Chi-square distribution with $n p$ degrees of freedom. Hence, $\chi_{n p, \alpha}^{2}$ which denotes the $1-\alpha$ percentile of a Chi-square distribution with $n p$ degrees of freedom can be used as an upper control limit for this statistic.

\section{MEWMA_3 control chart}

This approach is an extension of the approach given by Kim et al. (2003) where $x$ values are coded so that their average becomes zero. When $\bar{X}=0$, the covariance between the estimated slope and intercept of the profile becomes zero. In this situation, the $1 \times p$ vector of intercepts and $1 \times p$ vector of slopes are independent and can be monitored separately. Then, the monitoring process of regression parameters, as well as process variability, can be carried out using three separate control charts. In this case, the relation between the explanatory and response variables for the $i$ th observation is given by (Noorossana et al. 2010)

$\boldsymbol{y}_{i k}=\boldsymbol{\beta}_{0}^{\prime}+\boldsymbol{x}_{i}^{\prime} \boldsymbol{\beta}_{1}^{\prime}+\boldsymbol{\varepsilon}_{i k}, \quad i=1,2, \ldots, n$,

where $\boldsymbol{x}_{i}^{\prime}=\left(\boldsymbol{x}_{i}-\overline{\boldsymbol{x}}\right), \boldsymbol{\beta}_{1}^{\prime}=\boldsymbol{\beta}_{1}, \boldsymbol{\beta}_{1}^{\prime}=\boldsymbol{\beta}_{1}+\overline{\boldsymbol{x}} \boldsymbol{J}$ and $\boldsymbol{J}$ is a $1 \times p$ vector of 1's. In the new model, the estimation of intercepts is obtained by $\hat{\boldsymbol{\beta}}_{0 k}^{\prime}=\left(\bar{y}_{.1 k}, \bar{y}_{.2 k}, \ldots, \bar{y}_{. p k}\right)$ the $k$ th sample; however, the estimation of slopes is the same as the estimate in the original model. When the process is in-control, $\hat{\boldsymbol{\beta}}_{0 k}^{\prime}$ and $\hat{\boldsymbol{\beta}}_{1 k}^{\prime}$ follow a multivariate normal distribution with mean vectors of $\boldsymbol{\beta}_{0}^{\prime}$ and $\boldsymbol{\beta}_{1}^{\prime}$ and covariance matrices of $\boldsymbol{\Sigma}_{\boldsymbol{\beta}_{0}^{\prime}}=n^{-1} \boldsymbol{\Sigma}$ and $\boldsymbol{\Sigma}_{\boldsymbol{\beta}_{1}^{\prime}}=\left(S_{x x}\right)^{-1} \boldsymbol{\Sigma}$, respectively. Note that the covariance between each element of $\hat{\boldsymbol{\beta}}_{0 k}^{\prime}$ and $\hat{\boldsymbol{\beta}}_{1 k}^{\prime}$. is equal to zero. Hence, separate control charts for monitoring of $\boldsymbol{\beta}_{0}^{\prime}$ and $\boldsymbol{\beta}_{1}^{\prime}$ can be implemented (Noorossana et al. 2010).

The main advantage of using three separate control charts is easier identification of the parameter responsible for out-of-control signals. In the proposed MEWMA chart for monitoring of intercept vector $\left(\hat{\boldsymbol{\beta}}_{0 k}\right)$, the variable $z_{I k}$ can be computed by (Noorossana et al. 2010):

$z_{I k}=\theta\left(\hat{\boldsymbol{\beta}}_{0 k}^{\prime}-\boldsymbol{\beta}_{0}^{\prime}\right)+(1-\theta) z_{I(k-1)}, k=1,2, \ldots$,

where $z_{I 0}$ is a $1 \times p$ vector of zeros and $\theta(0<\theta \leq 1)$ is a smoothing parameter. It can be shown that when the process is in-control, $z_{I k}$ follows a $p$-variate normal distribution with an average vector of zero and a known covariance matrix $\boldsymbol{\Sigma}_{z I}=\left\{\frac{\theta}{n(2-\theta)}\right\} \boldsymbol{\Sigma}$. Therefore, the $T_{I k}^{2}$ statistic for the $k$ th sample is given as follows (Noorossana et al. 2010):

$T_{I k}^{2}=z_{I k} \Sigma_{z I}^{-1} z_{I k}^{T}, \quad k=1,2, \ldots$

The chart signals when $T_{I k}^{2}>h_{I}$ where $h_{I}$ is determined to achieve a specified in-control $A R L$.

In the MEWMA chart for slope monitoring, $z_{S k}$ can be calculated as follows (Noorossana et al. 2010):

$\boldsymbol{z}_{S k}=\theta\left(\hat{\boldsymbol{\beta}}_{1 k}^{\prime}-\boldsymbol{\beta}_{1}^{\prime}\right)+(1-\theta) \boldsymbol{z}_{S(k-1)}, k=1,2, \ldots$,

where $z_{S 0}$ is a $1 \times p$ vector of zeros and $\theta(0<\theta \leq 1)$ is a smoothing parameter. When the process is in-control, $z_{S k}$ follows a $p$-variate normal distribution with a mean vector of zero and a known covariance matrix $\boldsymbol{\Sigma}_{z S}=\left\{\frac{\theta}{(2-\theta)}\right\} \boldsymbol{\Sigma}_{\hat{\boldsymbol{\beta}}_{1}^{\prime}}=\left\{\frac{\theta}{S_{x x}(2-\theta)}\right\} \boldsymbol{\Sigma}$. Therefore, the MEWMA statistic for the $k$ th sample can be defined as follows (Noorossana et al. 2010):

$T_{S k}^{2}=z_{S k} \boldsymbol{\Sigma}_{z S}^{-1} z_{S k}^{T}, \quad k=1,2, \ldots$

The chart signals when $T_{S k}^{2}>h_{S}$ and $h_{S}$ is determined such that a specified in-control $A R L$ is obtained.

Another MEWMA control chart similar to that proposed by Crowder and Hamilton (1992) is developed for monitoring of process variability. The logarithm of the statistic $\chi_{k}^{2}=\sum_{i=1}^{n} \boldsymbol{e}_{i k} \boldsymbol{\Sigma}^{-1} \boldsymbol{e}_{i k}^{\mathrm{T}}$ is used for computing $\boldsymbol{z}_{E k}$ (Noorossana et al. 2010):

$z_{E k}=\operatorname{Max}\left\{\theta \ln \left(\chi_{k}^{2}\right)+(1-\theta) z_{E(k-1)}, n p\right\}, \quad k=1,2, \ldots$,

where $n p$ is the mean of a Chi-square distribution with $n p$ degrees of freedom and $\theta(0<\theta \leq 1)$ is a smoothing parameter. In this approach, the chart signals when $z_{E k}>h_{E}$ where $h_{E}$ is determined in such a way that a specified in-control $A R L$ is achieved. 


\section{The proposed approach for measuring the effect of parameter estimation on the performance of Phase II control charts}

Based on the literature, it is usually assumed that the parameters of a profile (matrix B) and the covariance matrix of the response variables $(\boldsymbol{\Sigma})$ are known in Phase II. In the current study, it is assumed that matrix $\mathbf{B}$ is unknown and should be estimated through in-control Phase I samples. Now the question is whether the $\mathbf{B}$ estimation affects the performance of the MSL profile monitoring approaches (MEWMA, MEWMA_3 and MEWMA $/ \chi^{2}$ methods) that have been developed based on known parameters, and which method is more robust to the effects of parameter estimation.

To answer these questions, a Monte Carlo simulation algorithm is proposed to evaluate the effect of parameter estimation on the performance of control chart schemes.

Note that the current study, for better comparison, uses the same multivariate profile model given by Noorossana et al. (2010):

$\left\{\begin{array}{l}Y_{1}=3+2 X+\varepsilon_{1} \\ Y_{2}=2+1 X+\varepsilon_{2} .\end{array}\right.$

Assume that $x_{i}$ values are set equal to 2, 4, 6 and $8(\bar{X}=5)$ which are considered to be fixed in all the sampling stages, and the covariance matrix of the response variables is $\boldsymbol{\Sigma}=\left[\begin{array}{ll}1 & 0.9 \\ 0.9 & 1\end{array}\right]$. The steps of the proposed Monte Carlo simulation algorithm are as follows:

1. Assume that $\mathbf{B}$ and $\boldsymbol{\Sigma}$ are known. Using 10,000 simulation runs under in-control conditions, calculate the upper control limits for each of the three methods to achieve $A R L_{0}=200$ when the parameters are known. The upper control limits for different values of $\theta$ are shown in Table 1. Note that Noorossana et al. (2010) obtained the upper control limits for each of the three methods just for $\theta=0.2$. In the current study, the control limits were obtained for $\theta=0.05,0.1$ to evaluate the effect of $\theta$ on the control limit values. Table 1 shows that the control limit values increase when $\theta$ increases. It should be noted that since the $\chi^{2}$ statistic is not dependent on $\theta$, this parameter does not affect the upper limit of the $\chi^{2}$ control chart.

2. Generate $m$ in-control MSL profiles based on known parameters using a multivariate normal distribution with a mean vector of zero and a covariance matrix $\boldsymbol{\Sigma}$.

3. Calculate $\hat{\mathbf{B}}_{k}$ where $k=1,2, \ldots, m$ for each of the profiles generated in step 2 , then calculate $\hat{\hat{\mathbf{B}}}=\frac{\sum_{k=1}^{m} \hat{\mathbf{B}}_{k}}{m}$.

4. Generate an in-control profile based on known parameters and estimate the matrix of profile parameters $(\mathbf{B})$
Table 1 In-control upper control limits of three control charts approaches

\begin{tabular}{|c|c|c|c|c|c|}
\hline \multirow{3}{*}{$\begin{array}{l}\theta \\
0.05\end{array}$} & \multicolumn{5}{|c|}{ Control chart } \\
\hline & \multirow{2}{*}{$\begin{array}{l}\text { MEWMA } \\
11.2\end{array}$} & \multicolumn{2}{|c|}{ MEWMA $/ \chi^{2}$} & \multicolumn{2}{|l|}{ MEWMA_3 } \\
\hline & & MEWMA & 9.07 & MEWMA $_{(I)}$ & 10.68 \\
\hline & & & & $\operatorname{MEWMA}_{(\mathrm{S})}$ & 10.68 \\
\hline & & $\chi^{2}$ & 23.77 & $\operatorname{MEWMA}_{(\mathrm{E})}$ & 2.208 \\
\hline \multirow[t]{3}{*}{0.1} & 12.7 & MEWMA & 10.25 & $\mathrm{MEWMA}_{(\mathrm{I})}$ & 11.82 \\
\hline & & & & $\operatorname{MEWMA}_{(\mathrm{S})}$ & 11.82 \\
\hline & & $\chi^{2}$ & 23.77 & $\operatorname{MEWMA}_{(\mathrm{E})}$ & 2.298 \\
\hline \multirow[t]{3}{*}{0.2} & 13.9 & MEWMA & 11.1 & $\operatorname{MEWMA}_{(\mathrm{I})}$ & 12.55 \\
\hline & & & & $\operatorname{MEWMA}_{(\mathrm{S})}$ & 12.55 \\
\hline & & $\chi^{2}$ & 23.77 & $\operatorname{MEWMA}_{(\mathrm{E})}$ & 2.43 \\
\hline
\end{tabular}

using Eq. 5, and calculate the chart statistic. Note that $\hat{\overline{\mathbf{B}}}$ obtained in step 3 should be considered as the estimation of profile parameters, instead of $\mathbf{B}$ which contains known parameters. Then, set $R L=1$.

5. Compare the calculated chart statistic with the corresponding upper control limit (UCL) (Table 1). If the chart statistic is larger than the $U C L$, go to step 6; otherwise, set $R L=R L+1$ and go to step 4 .

6. Record the $R L$ value and go to step 4 .

7. Repeat steps 4-6, 10,000 times and calculate the $A R L$ by averaging the $R L$ values. Then, go to step 2 .

8. Repeat steps $2-7,10,000$ times to achieve 10,000 different values of the $A R L$. Then, calculate the $A A R L=\operatorname{Mean}(A R L), S D A R L=S T D(A R L)$ and $C V A R L$ using Eq. 1.

\section{Comparison of control chart performance under in-control conditions}

In this section, the in-control performance of the three control chart approaches for monitoring MSL profiles is compared when profile parameters are estimated using the simulation algorithm described in "The proposed approach for measuring the effect of parameter estimation on the performance of phase II control charts" section. Table 2 contains the results of the simulation study for different values of $m$ and $\theta$ in order to compare the performance of the control chart schemes in terms of the in-control AARL, SDARL and CVARL metrics. In general, more Phase I samples lead to better estimation that is closer to the desired in-control $A A R L=200, S D A R L=0$ and $C V A R L=0$.

Since three different metrics were used in the current study, it is better to separately compare the performance of the schemes in terms of each metric. Table 2 and Fig. 1 
Table 2 In-Control AARL, SDARL and CVARL comparisons of MEWMA, MEWMA/ $\chi^{2}$ and MEWMA_3 methods when $m$ samples of Phase I are used to estimate the unknown parameters

\begin{tabular}{|c|c|c|c|c|c|c|c|c|c|}
\hline \multirow[t]{2}{*}{$m$} & \multicolumn{3}{|l|}{$A A R L$} & \multicolumn{3}{|l|}{$S D A R L$} & \multicolumn{3}{|l|}{ CVARL $(\%)$} \\
\hline & MEWMA & MEWMA $/ \chi^{2}$ & MEWMA_3 & MEWMA & MEWMA $/ \chi^{2}$ & MEWMA_3 & MEWMA & MEWMA $/ \chi^{2}$ & MEWMA_3 \\
\hline \multicolumn{10}{|l|}{$\theta=0.2$} \\
\hline 10 & 43.8 & 85.08 & 57.9 & 42.64 & 47.86 & 39.85 & 97.35 & 56.24 & 68.82 \\
\hline 70 & 132.56 & 155.2 & 142.96 & 34.93 & 32.93 & 29.25 & 26.35 & 21.22 & 20.44 \\
\hline 200 & 166.53 & 180.57 & 179.35 & 20.69 & 18.24 & 15.31 & 12.42 & 10.1 & 8.5 \\
\hline 500 & 185.53 & 194.99 & 191.81 & 12.43 & 8.6 & 9.3 & 6.70 & 4.42 & 4.8 \\
\hline 1000 & 194.79 & 197.95 & 198.05 & 7.32 & 6.04 & 7.78 & 3.76 & 3.05 & 3.9 \\
\hline 2000 & 200.07 & 201.19 & 199.68 & 6.2 & 5.9 & 6.63 & 3.10 & 2.9 & 3.32 \\
\hline \multicolumn{10}{|l|}{$\theta=0.1$} \\
\hline 10 & 35.72 & 70.27 & 44.83 & 37.654 & 46.33 & 38.65 & 105.41 & 65.93 & 86.21 \\
\hline 70 & 105.36 & 139.58 & 128.54 & 34.54 & 40.22 & 36.9 & 32.78 & 28.82 & 28.71 \\
\hline 200 & 149.22 & 174.57 & 179.13 & 25.75 & 25.33 & 19.48 & 17.26 & 14.51 & 10.87 \\
\hline 500 & 175.19 & 195.51 & 195.82 & 16.22 & 11.5 & 13.04 & 9.26 & 5.88 & 6.66 \\
\hline 1000 & 186.89 & 197.63 & 206 & 9.48 & 10.24 & 8.15 & 5.07 & 5.18 & 3.96 \\
\hline 2000 & 191.36 & 202.06 & 201.33 & 7.46 & 6.65 & 7.75 & 3.90 & 3.29 & 3.85 \\
\hline \multicolumn{10}{|l|}{$\theta=0.05$} \\
\hline 10 & 32.35 & 54.84 & 39.83 & 36.47 & 48.64 & 39.11 & 112.74 & 88.69 & 98.19 \\
\hline 70 & 90.72 & 131.03 & 116.51 & 32.32 & 42.26 & 35.89 & 35.63 & 32.25 & 30.80 \\
\hline 200 & 143.39 & 168 & 161.81 & 30.14 & 27.42 & 26.28 & 21.02 & 16.32 & 16.24 \\
\hline 500 & 169.68 & 188.31 & 186.77 & 15.76 & 16.04 & 15.98 & 9.29 & 8.52 & 8.56 \\
\hline 1000 & 181.53 & 198.32 & 200.45 & 12.33 & 10.51 & 10.24 & 6.79 & 5.30 & 5.11 \\
\hline 2000 & 193.68 & 203.82 & 205.63 & 6.6 & 8.05 & 8.16 & 3.41 & 3.95 & 3.97 \\
\hline
\end{tabular}

show that the performance of the control chart approaches under in-control conditions is strongly affected by parameter estimation. Based on the $A A R L$ metric, it can be inferred that the MEWMA $/ \chi^{2}$ method performs better than competing methods because of a larger $A A R L$ value in most of the cases. For instance, if $\theta=0.2$, the $A A R L$ value of the MEWMA $/ \chi^{2}$ method is almost $90 \%$ of the specified in-control $A R L\left(A R L_{0}=200\right)$ using a smaller number of samples ( $m=200)$ than the other methods.

Table 1 shows that the AARL value increases when the value of the smoothing parameter $(\theta)$ increases. This could be very useful in practical environments. In some industries, such as military or high-tech industries, a high number of false alarms lead to high expenses; therefore, choosing the method that has a larger in-control $A A R L$ is suggested in order to reduce the rate of false alarms.

Figure 2 shows a comparison of the performance of the control chart schemes in terms of the SDARL metric for different values of $m$ and $\theta$. It is obvious that smaller values of the $S D A R L$ represent better performance of the control chart schemes. However, it is recommended that the $S D A R L$ be within $5-10 \%$ of the desired in-control $A R L$. Choosing the superior method in terms of the SDARL metric is not clear in this case. According to Fig. 2, there is no method that results in smaller $S D A R L$ value than the other competing methods for all the values of $m$. This ambiguity rises when the value of $\theta$ decreases. However, it can be confidently inferred that the MEWMA_3 method performs best in terms of the SDARL except for small values of $m(m=10)$ when $\theta=0.2$. Furthermore, the MEWMA_3 and MEWMA $/ \chi^{2}$ methods reached SDARL values that are within 5\%-10\% of the desired in-control $A R L=200$ using a smaller number of Phase I samples than the MEWMA method. Now the question is: What is the best decision in such situations?

The solution we propose is using the CVARL metric, which is calculated by Eq. 1. Using this metric allows both the $A A R L$ and $S D A R L$ to be considered simultaneously in deciding which is the superior method. Aly et al. (2016) used this metric in their study on adaptive EWMA control charts. Figure 3 shows the CVARL comparison of the control chart schemes when the process is in-control. Whereas the MEWMA_3 and MEWMA $/ \chi^{2}$ methods both perform better than the MWEMA method, the MEWMA_3 method uniformly performs better than the other competing methods. Moreover, the performance of the MEWMA_3 method improves as the value of $m$ increases. 


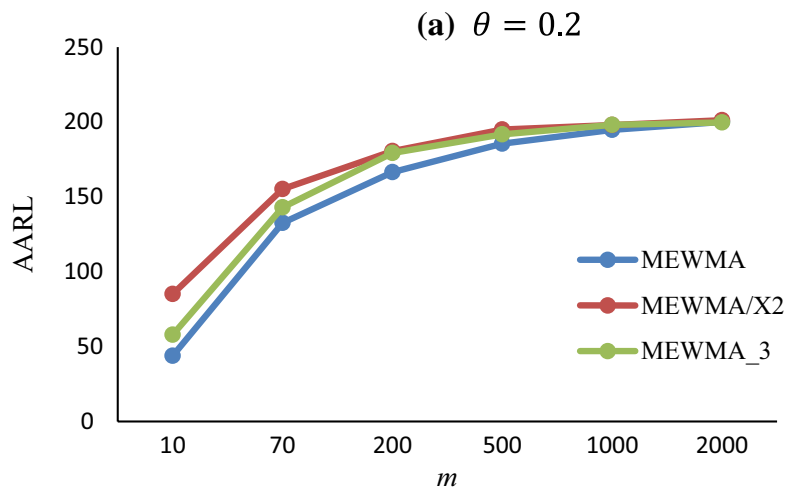

(b) $\theta=0.1$
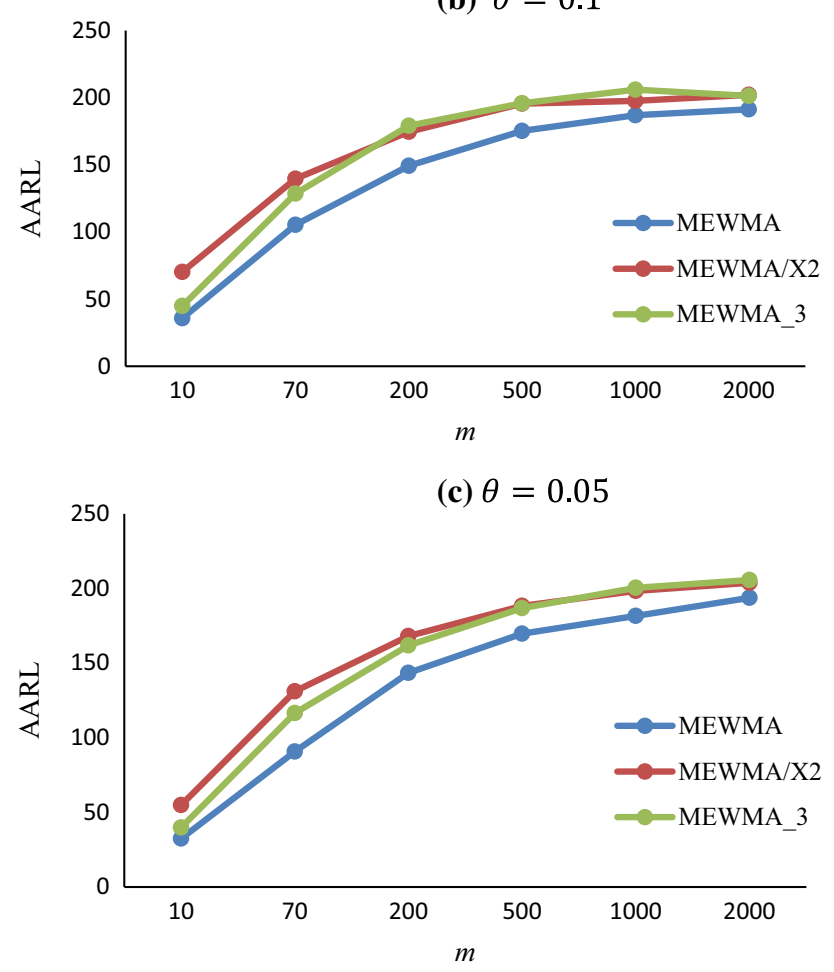

Fig. 1 AARL comparison of three control charts schemes for $m=10$, $70,200,500,1000,2000$ and $\theta=0.2,0.1,0.05$

\section{The proposed approach for establishing corrected limits}

As observed in "Comparison of control chart performance under in-control conditions" section, in the MEWMA/ $\chi^{2}$ method, we need at least 200 in-control Phase I samples for parameters estimation so that the $A A R L$ value is at least $90 \%$ of the specified in-control $A R L_{0}=200$. Therefore, the practitioner should wait too long to collect samples. Hence, in these situations, using corrected control limits is strongly suggested. By using corrected limits which are usually wider than original ones due to the extra variability added to the process by estimators, the problem of large Phase I samples requirement for estimation can be solved. Corrected limits

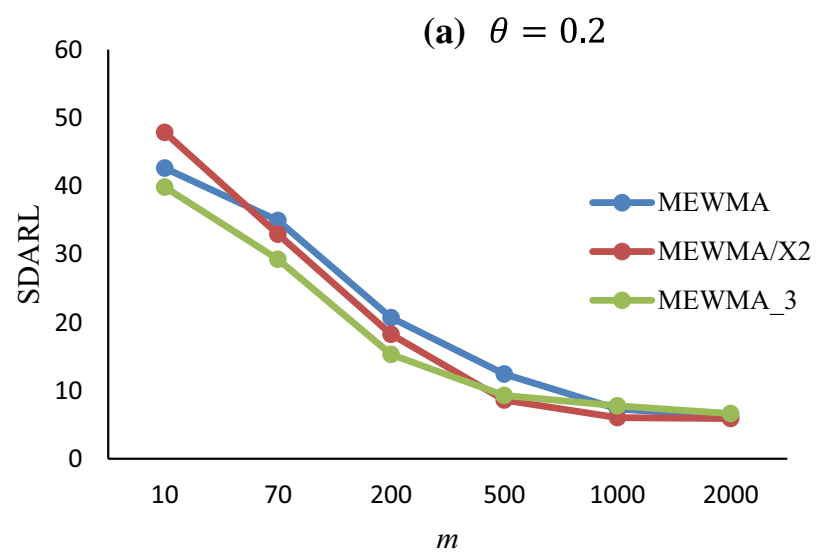

(b) $\theta=0.1$
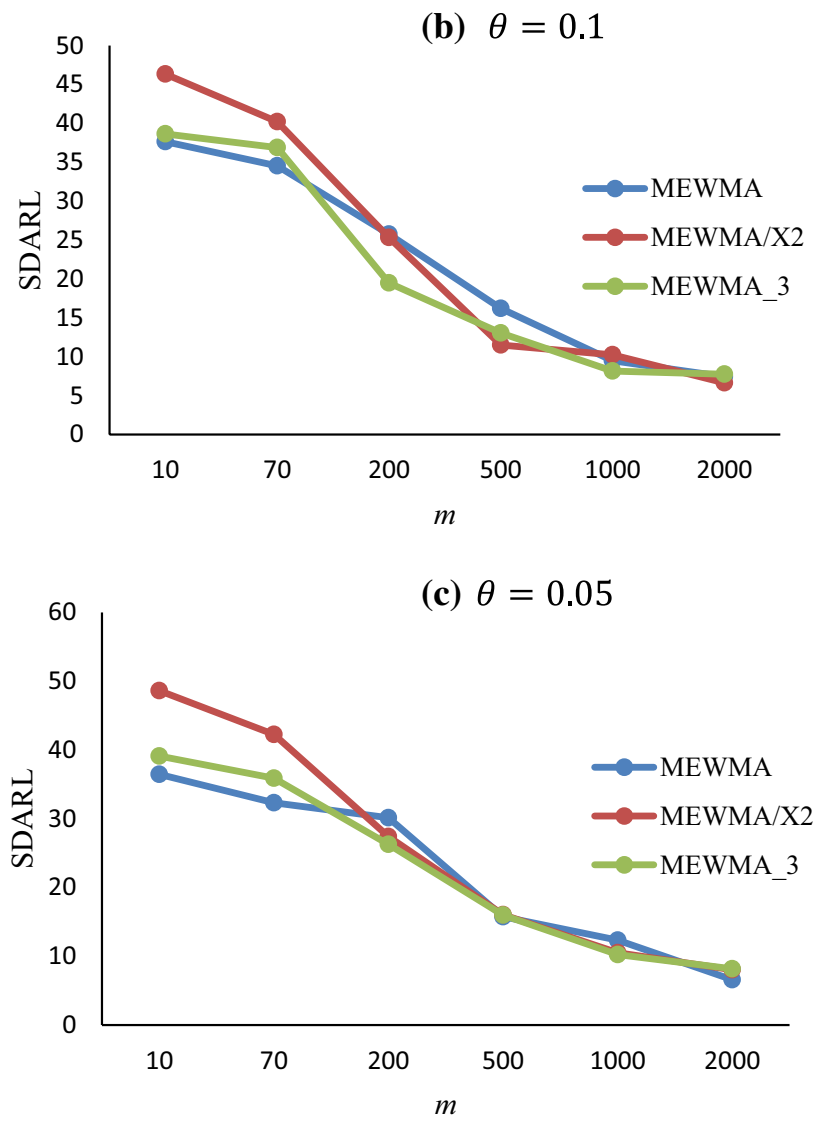

Fig. 2 SDARL comparison of three control charts schemes for $m=10,70,200,500,1000,2000$ and $\theta=0.2,0.1,0.05$

can also be established based on the small size of historical data set. However, it should be noted that very wide corrected limits due to very small Phase I samples can deteriorate the detection performance of Phase II control charts. To learn more about corrected limits please see Quesenberry (1993), Jones (2002), Champ et al. (2005) and Mahmoud and Maravelakis (2010).

In the current study, the corrected limits are obtained through the Monte Carlo simulation algorithm discussed in 

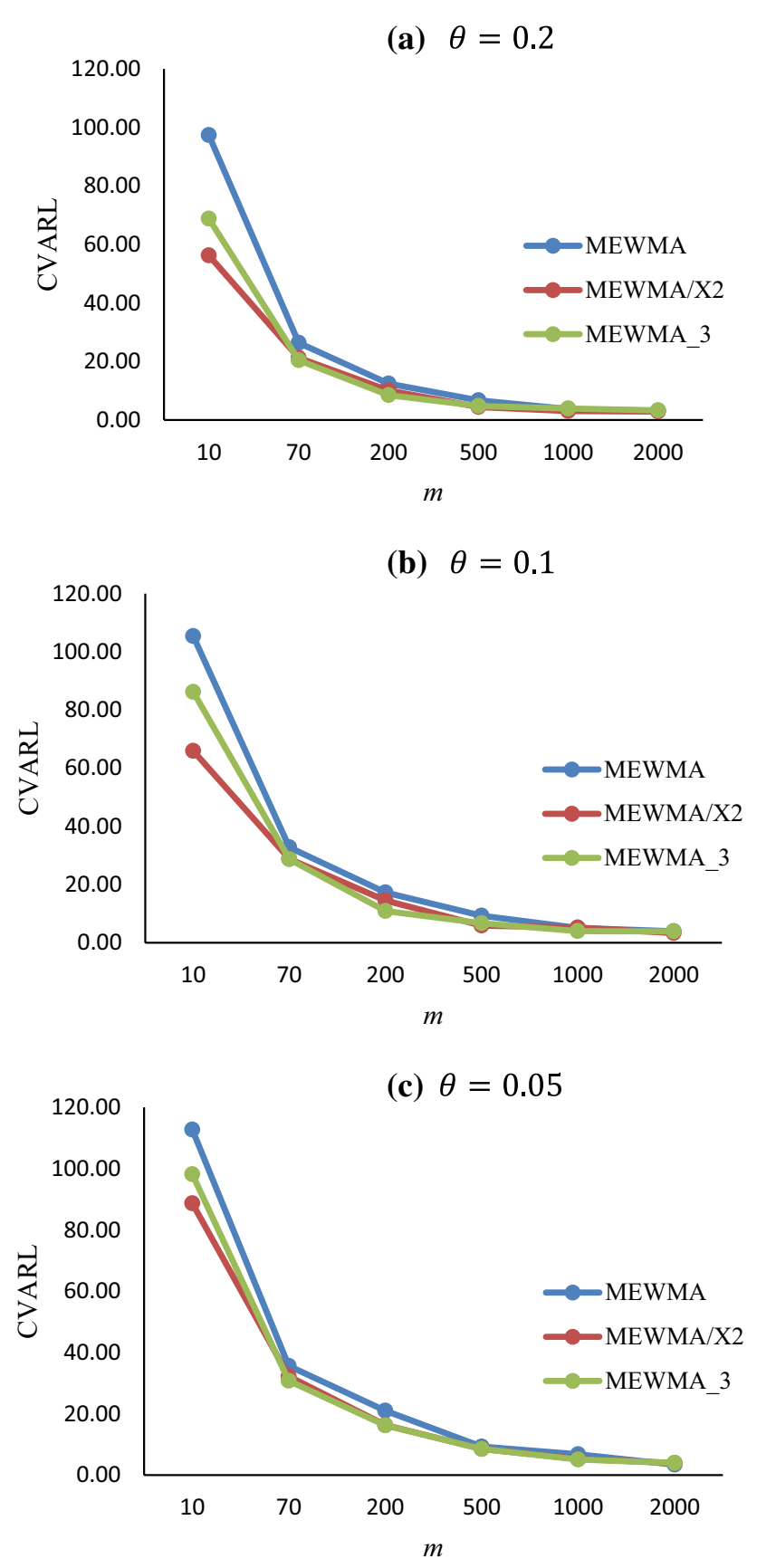

Fig. 3 CVARL comparison of three control charts schemes for $m=10,70,200,500,1000,2000$ and $\theta=0.2,0.1,0.05$

"The proposed approach for measuring the effect of parameter estimation on the performance of Phase II control charts" section for each of the profile monitoring methods to achieve $A A R L \cong 200$ by 10,000 simulation runs and using the number of $m$ in-control Phase I profiles for parameter estimation. Note that in the previous studies, such as Mahmoud (2012), the corrected limits are obtained to achieve a specified incontrol $A R L$. However, in the current study, the corrected limits are obtained based on achieving in-control $A A R L$ in order to consider practitioner-to-practitioner variability. The results of the simulation study are shown in Table 3 . The corrected limits are usually wider than the control limits derived based on known parameters (Table 1). Therefore, the control limits given in Table 1 can be used as the starting point in simulation runs. Similar to the known parameter case, corrected limits also increase when $\theta$ increases.

Corrected limits could be used in evaluating the out-ofcontrol performance of control charts. Assume that when parameters are estimated, a shift has occurred in one of the profile parameters and the control chart has alarmed. In this situation, we cannot determine whether this alarm is due to the effect of parameter estimation or the effect of the shift in the in-control parameter. Hence, using corrected limits ensures that chart alarming occurs only because of the shift in profile parameters. Consequently, evaluating the performance of the control chart schemes under out-of-control conditions was carried out using corrected limits; the results are shown in Table 3.

\section{Comparison of control chart performance under out-of-control conditions}

Several types of shifts are considered to evaluate the outof-control performance of control chart schemes using parameter estimation, including: (1) a shift in the intercept of the first profile $\left(\beta_{01}\right) ;(2)$ a shift in the slope of the first profile $\left(\beta_{11}\right)$. To apply these shifts, we should generate an out-of-control profile based on the shifted parameters in step 4 of the simulation algorithm presented in "The proposed approach for measuring the effect of parameter estimation on the performance of Phase II control charts" section.

Note that the applied shifts in this paper are the same as the shifts applied in a study by Noorossana et al. (2010), which was based on known parameters, in order to compare the results of our study (when parameters are estimated) with the results of Noorossana et al. (2010) (when parameters are known). In addition, the corrected limits (Table 3) are used to compare the out-of-control performance of the control chart schemes.

Table 4 shows the out-of-control AARL, SDARL and $C V A R L$ metrics of the control chart schemes when $\beta_{01}$ shifts to $\beta_{01}+\tau_{0} \sigma_{1}$. In this case, the performance of the MEWMA and MEWMA $/ \chi^{2}$ methods is uniformly better than that of the MEWMA_3 method in terms of the AARL for all values of $m$. However, the performance of the

MEWMA $/ \chi^{2}$ method is better than the MEWMA method for small shifts; for the large shifts, both methods perform similarly. On the other hand, the MEWMA method has a smaller SDARL value than the other competing methods. However, the SDARL performance of MEWMA and MEWMA $/ \chi^{2}$ methods are almost similar. 
Table 3 Corrected limits for three control charts in order to achieve $A A R L=200$ for different values of $m$ and $\theta$

\begin{tabular}{|c|c|c|c|c|c|}
\hline \multirow{2}{*}{$\frac{m}{\theta=0.2}$} & \multirow[t]{2}{*}{ MEWMA } & \multicolumn{2}{|c|}{ MEWMA $/ \chi^{2}$} & \multicolumn{2}{|l|}{ MEWMA_3 } \\
\hline & & & & & \\
\hline \multirow[t]{3}{*}{30} & 16.9 & MEWMA & 12.76 & $\mathrm{MEWMA}_{(\mathrm{I})}$ & 14.13 \\
\hline & & & & $\operatorname{MEWMA}_{(\mathrm{S})}$ & 14.1 \\
\hline & & $\chi^{2}$ & 27.62 & $\mathrm{MEWMA}_{(\mathrm{E})}$ & 4.12 \\
\hline \multirow[t]{3}{*}{50} & 15.5 & MEWMA & 12.12 & MEWMA $_{(\mathrm{I})}$ & 13.71 \\
\hline & & & & $\mathrm{MEWMA}_{(\mathrm{S})}$ & 13.64 \\
\hline & & $\chi^{2}$ & $26.86 \mathrm{M}$ & $\mathrm{MEWMA}_{(\mathrm{E})}$ & 3.82 \\
\hline \multirow[t]{3}{*}{100} & 14.9 & MEWMA & 12.21 & MEWMA $_{(\mathrm{I})}$ & 13.33 \\
\hline & & & & $\operatorname{MEWMA}_{(\mathrm{S})}$ & 13.2 \\
\hline & & $\chi^{2}$ & 24.91 & $\mathrm{MEWMA}_{(\mathrm{E})}$ & 3.22 \\
\hline \multirow[t]{3}{*}{300} & 14.2 & MEWMA & 12.24 & MEWMA $_{(\mathrm{I})}$ & 13.19 \\
\hline & & & & $\operatorname{MEWMA}_{(\mathrm{S})}$ & 12.94 \\
\hline & & $\chi^{2}$ & 24.35 & $\mathrm{MEWMA}_{(\mathrm{E})}$ & 2.9 \\
\hline \multirow[t]{3}{*}{600} & 14.05 & MEWMA & 11.73 & MEWMA $_{(\mathrm{I})}$ & 12.91 \\
\hline & & & & $\operatorname{MEWMA}_{(\mathrm{S})}$ & 12.83 \\
\hline & & $\chi^{2}$ & 24.1 & $\operatorname{MEWMA}_{(\mathrm{E})}$ & 2.68 \\
\hline \multicolumn{6}{|l|}{$\theta=0.1$} \\
\hline \multirow[t]{3}{*}{30} & 16.52 & MEWMA & 12.6 & MEWMA $_{(\mathrm{I})}$ & 13.45 \\
\hline & & & & $\operatorname{MEWMA}_{(\mathrm{S})}$ & 13.81 \\
\hline & & $\chi^{2}$ & 27.62 & $\operatorname{MEWMA}_{(\mathrm{E})}$ & 3.515 \\
\hline \multirow[t]{3}{*}{50} & 15.3 & MEWMA & 11.85 & MEWMA $_{(\mathrm{I})}$ & 13.18 \\
\hline & & & & $\operatorname{MEWMA}_{(\mathrm{S})}$ & 13.11 \\
\hline & & $\chi^{2}$ & 26.86 & $\operatorname{MEWMA}_{(\mathrm{E})}$ & 3.322 \\
\hline \multirow[t]{3}{*}{100} & 14.1 & MEWMA & 11.5 & MEWMA $_{(\mathrm{I})}$ & 12.83 \\
\hline & & & & $\operatorname{MEWMA}_{(\mathrm{S})}$ & 12.75 \\
\hline & & $\chi^{2}$ & 24.91 & $\operatorname{MEWMA}_{(\mathrm{E})}$ & 3.032 \\
\hline \multirow[t]{3}{*}{300} & 13.4 & MEWMA & 10.75 & $\operatorname{MEWMA}_{(\mathrm{I})}$ & 12.32 \\
\hline & & & & $\operatorname{MEWMA}_{(\mathrm{S})}$ & 12.44 \\
\hline & & $\chi^{2}$ & 24.35 & $\operatorname{MEWMA}_{(\mathrm{E})}$ & 2.882 \\
\hline \multirow[t]{3}{*}{600} & 13.05 & MEWMA & 10.55 & $\mathrm{MEWMA}_{(\mathrm{I})}$ & 12.18 \\
\hline & & & & $\operatorname{MEWMA}_{(\mathrm{S})}$ & 12.11 \\
\hline & & $\chi^{2}$ & 24.1 & MEWMA $_{(\mathrm{E})}$ & 2.544 \\
\hline \multicolumn{6}{|c|}{$\theta=0.05$} \\
\hline \multirow[t]{3}{*}{30} & 16.25 & MEWMA & 11.44 & MEWMA $_{(\mathrm{I})}$ & 13.42 \\
\hline & & & & $\operatorname{MEWMA}_{(\mathrm{S})}$ & 13.88 \\
\hline & & $\chi^{2}$ & 27.62 & $\operatorname{MEWMA}_{(\mathrm{E})}$ & 3.201 \\
\hline \multirow[t]{3}{*}{50} & 15.1 & MEWMA & 10.93 & MEWMA $_{(\mathrm{I})}$ & 13.19 \\
\hline & & & & $\operatorname{MEWMA}_{(\mathrm{S})}$ & 13.46 \\
\hline & & $\chi^{2}$ & 26.86 & $\operatorname{MEWMA}_{(\mathrm{E})}$ & 2.881 \\
\hline \multirow[t]{3}{*}{100} & 13.4 & MEWMA & 10.54 & $\operatorname{MEWMA}_{(\mathrm{I})}$ & 12.87 \\
\hline & & & & $\operatorname{MEWMA}_{(\mathrm{S})}$ & 12.65 \\
\hline & & $\chi^{2}$ & 24.91 & $\operatorname{MEWMA}_{(\mathrm{E})}$ & 2.621 \\
\hline \multirow[t]{3}{*}{300} & 12.1 & MEWMA & 9.92 & $\operatorname{MEWMA}_{(\mathrm{I})}$ & 11.83 \\
\hline & & & & $\operatorname{MEWMA}_{(\mathrm{S})}$ & 11.92 \\
\hline & & $\chi^{2}$ & 24.35 & $\operatorname{MEWMA}_{(\mathrm{E})}$ & 2.411 \\
\hline \multirow[t]{3}{*}{600} & 11.7 & MEWMA & 9.47 & $\operatorname{MEWMA}_{(\mathrm{I})}$ & 11.48 \\
\hline & & & & $\operatorname{MEWMA}_{(\mathrm{S})}$ & 11.34 \\
\hline & & $\chi^{2}$ & 24.1 & $\operatorname{MEWMA}_{(\mathrm{E})}$ & 2.369 \\
\hline
\end{tabular}

Finally, considering the CVARL metric, showed better performance of the MEWMA method compared with the other competing methods. However, Noorossana et al. (2010) found that the MEWMA $/ \chi^{2}$ method performs better compared to the other competing methods in terms of outof-control $A R L$ when the parameters are known.

The out-of-control AARL, SDARL and CVARL values when $\beta_{11}$ shifts to $\beta_{11}+\tau_{1} \sigma_{1}$ are given in Table 5. Similar to the case in which the parameters are known, the results show that the MEWMA method performs better than the other competing methods in terms of all three metrics. However, the MEWMA $/ \chi^{2}$ method is more capable of detecting very small shifts than the other methods in terms of the CVARL.

Based on the results obtained, it is possible to conclude that control chart performance under out-of-control conditions is strongly affected by the parameter estimation, similar to in-control conditions. When estimated parameters are used for calculating the control chart statistic, a large number of Phase I samples are needed in order to achieve the same in-control performance as when the parameters are known. On the other hand, taking more Phase I samples for the parameter estimation leads to better detection performance.

Therefore, we can say with confidence not only that the in-control and out-of-control performance of control chart schemes for monitoring MSL profiles is seriously affected by parameter estimation, but also that ignoring the variability added to the process by parameter estimation can affect choosing the superior monitoring scheme.

This could have many economic and competitive advantages in manufacturing and non-manufacturing industries because of decreasing the rate of false alarms.

\section{Conclusion and suggestions for future research}

The current study was an investigation of the effect of parameter estimation on three approaches to MSL profile monitoring. The approaches are: (1) a MEWMA control chart for monitoring profile parameters; (2) a combination of a MEWMA control chart based on the vector of residual means and a $\chi^{2}$ chart for monitoring process variability (MEWMA $/ \chi^{2}$ ); and (3) a combination of three separate MEWMA control charts (MEWMA_3) for monitoring the vector of intercepts, the vector of slopes and the process variability. Since the $A R L$ is no longer a parameter when it is assumed that the process parameters are estimated, we used three metrics, the AARL, SDARL and CVARL, which are based on the statistical properties of the $A R L$ distribution. Our goal is to compare the methods and choose the best one, which is more robust to the effect of parameter estimation. The simulation results showed that the MEWMA/ $\chi^{2}$ method performs better in terms of the in-control AARL 
Table 4 AARL, SDARL and $C V A R L$ comparison when $\beta_{01}$ has shifted to $\beta_{01}+\tau_{0} \sigma_{1}$

\begin{tabular}{|c|c|c|c|c|c|c|c|c|c|c|c|c|}
\hline \multirow[t]{2}{*}{$m$} & \multirow[t]{2}{*}{ Method } & \multirow[t]{2}{*}{ Metric } & \multicolumn{10}{|l|}{$\tau_{0}$} \\
\hline & & & 0.2 & 0.4 & 0.6 & 0.8 & 1 & 1.2 & 1.4 & 1.6 & 1.8 & 2 \\
\hline \multirow[t]{9}{*}{30} & \multirow[t]{3}{*}{ MEWMA } & $A A R L$ & 18.43 & 5.31 & 3.26 & 2.54 & 1.98 & 1.91 & 1.54 & 1.20 & 1.02 & 1.00 \\
\hline & & $S D A R L$ & 7.43 & 0.49 & 0.28 & 0.09 & 0.07 & 0.04 & 0.05 & 0.03 & 0.01 & 0.00 \\
\hline & & CVARL & 40.31 & 9.23 & 8.59 & 3.54 & 3.54 & 2.09 & 3.25 & 2.50 & 0.98 & 0.00 \\
\hline & \multirow[t]{3}{*}{ MEWMA_3 } & $A A R L$ & 21.36 & 6.11 & 3.40 & 2.57 & 2.03 & 1.93 & 1.54 & 1.23 & 1.02 & 1.08 \\
\hline & & $S D A R L$ & 5.42 & 0.63 & 0.28 & 0.19 & 0.13 & 0.07 & 0.05 & 0.03 & 0.01 & 0.01 \\
\hline & & CVARL & 25.37 & 10.31 & 8.24 & 7.39 & 6.40 & 3.63 & 3.25 & 2.44 & 0.98 & 0.93 \\
\hline & \multirow[t]{3}{*}{ MEWMA $/ \chi^{2}$} & $A A R L$ & 17.64 & 5.12 & 2.93 & 1.98 & 1.55 & 1.42 & 1.21 & 1.07 & 1.01 & 1.00 \\
\hline & & $S D A R L$ & 9.24 & 0.62 & 0.33 & 0.11 & 0.09 & 0.05 & 0.05 & 0.03 & 0.01 & 0.00 \\
\hline & & CVARL & 52.38 & 12.11 & 11.26 & 5.56 & 5.81 & 3.52 & 4.13 & 2.80 & 0.99 & 0.00 \\
\hline \multirow[t]{9}{*}{50} & \multirow[t]{3}{*}{ MEWMA } & $A A R L$ & 17.53 & 5.26 & 3.21 & 2.43 & 1.97 & 1.91 & 1.52 & 1.18 & 1.08 & 1.00 \\
\hline & & $S D A R L$ & 4.70 & 0.48 & 0.18 & 0.07 & 0.07 & 0.04 & 0.04 & 0.03 & 0.01 & 0.00 \\
\hline & & CVARL & 26.81 & 9.13 & 5.61 & 2.88 & 3.55 & 2.09 & 2.63 & 2.54 & 0.93 & 0.00 \\
\hline & \multirow[t]{3}{*}{ MEWMA_3 } & $A A R L$ & 21.73 & 5.41 & 3.36 & 2.52 & 2.16 & 1.86 & 1.50 & 1.20 & 1.12 & 1.06 \\
\hline & & $S D A R L$ & 5.41 & 0.53 & 0.26 & 0.11 & 0.08 & 0.08 & 0.06 & 0.05 & 0.03 & 0.01 \\
\hline & & CVARL & 24.90 & 9.80 & 7.74 & 4.37 & 3.70 & 4.30 & 4.00 & 4.17 & 2.68 & 0.94 \\
\hline & \multirow[t]{3}{*}{ MEWMA $/ \chi^{2}$} & $A A R L$ & 16.11 & 5.03 & 2.87 & 1.98 & 1.54 & 1.41 & 1.18 & 1.07 & 1.01 & 1.00 \\
\hline & & $S D A R L$ & 5.34 & 0.51 & 0.20 & 0.08 & 0.06 & 0.05 & 0.05 & 0.03 & 0.01 & 0.00 \\
\hline & & CVARL & 33.15 & 10.14 & 6.97 & 4.04 & 3.90 & 3.55 & 4.24 & 2.80 & 0.99 & 0.00 \\
\hline \multirow[t]{9}{*}{100} & \multirow[t]{3}{*}{ MEWMA } & $A A R L$ & 17.41 & 5.25 & 3.19 & 2.41 & 1.94 & 1.87 & 1.49 & 1.16 & 1.08 & 1.00 \\
\hline & & $S D A R L$ & 2.73 & 0.27 & 0.13 & 0.04 & 0.04 & 0.03 & 0.03 & 0.02 & 0.01 & 0.00 \\
\hline & & $C V A R L$ & 15.68 & 5.14 & 4.08 & 1.66 & 2.06 & 1.60 & 2.01 & 1.72 & 0.93 & 0.00 \\
\hline & \multirow[t]{3}{*}{ MEWMA_3 } & $A A R L$ & 21.44 & 5.34 & 3.34 & 2.51 & 2.16 & 1.86 & 1.51 & 1.18 & 1.10 & 1.03 \\
\hline & & $S D A R L$ & 2.84 & 0.34 & 0.19 & 0.14 & 0.07 & 0.06 & 0.04 & 0.04 & 0.03 & 0.01 \\
\hline & & CVARL & 13.25 & 6.37 & 5.69 & 5.58 & 3.24 & 3.23 & 2.65 & 3.39 & 2.73 & 0.97 \\
\hline & \multirow[t]{3}{*}{ MEWMA $/ \chi^{2}$} & $A A R L$ & 15.34 & 4.88 & 2.43 & 1.94 & 1.48 & 1.36 & 1.15 & 1.05 & 1.00 & 1.00 \\
\hline & & $S D A R L$ & 2.76 & 0.36 & 0.16 & 0.08 & 0.05 & 0.05 & 0.04 & 0.02 & 0.00 & 0.00 \\
\hline & & CVARL & 17.99 & 7.38 & 6.58 & 4.12 & 3.38 & 3.68 & 3.48 & 1.90 & 0.00 & 0.00 \\
\hline \multirow[t]{9}{*}{300} & \multirow[t]{3}{*}{ MEWMA } & $A A R L$ & 17.46 & 5.23 & 3.17 & 2.37 & 1.97 & 1.83 & 1.43 & 1.16 & 1.06 & 1.00 \\
\hline & & $S D A R L$ & 2.59 & 0.25 & 0.11 & 0.03 & 0.02 & 0.02 & 0.01 & 0.02 & 0.01 & 0.00 \\
\hline & & CVARL & 14.85 & 4.70 & 3.47 & 1.47 & 0.83 & 1.09 & 0.70 & 1.72 & 0.94 & 0.00 \\
\hline & MEWMA_3 & $A A R L$ & 19.76 & 5.34 & 3.31 & 2.46 & 2.14 & 1.86 & 1.47 & 1.16 & 1.08 & 1.00 \\
\hline & & $S D A R L$ & 1.83 & 0.34 & 0.18 & 0.10 & 0.07 & 0.05 & 0.05 & 0.04 & 0.02 & 0.00 \\
\hline & & $C V A R L$ & 9.26 & 6.37 & 5.44 & 4.07 & 3.27 & 2.69 & 3.40 & 3.45 & 1.85 & 0.00 \\
\hline & MEWMA $/ \chi^{2}$ & $A A R L$ & 15.37 & 4.86 & 2.39 & 1.92 & 1.43 & 1.31 & 1.15 & 1.03 & 1.00 & 1.00 \\
\hline & & $S D A R L$ & 2.63 & 0.27 & 0.15 & 0.08 & 0.04 & 0.04 & 0.03 & 0.00 & 0.00 & 0.00 \\
\hline & & $C V A R L$ & 17.11 & 5.56 & 6.28 & 4.17 & 2.80 & 3.05 & 2.61 & 0.00 & 0.00 & 0.00 \\
\hline 600 & MEWMA & $A A R L$ & 17.24 & 5.14 & 3.23 & 2.37 & 1.99 & 1.73 & 1.40 & 1.12 & 1.02 & 1.00 \\
\hline & & $S D A R L$ & 1.31 & 0.19 & 0.04 & 0.02 & 0.02 & 0.02 & 0.02 & 0.02 & 0.01 & 0.00 \\
\hline & & CVARL & 7.60 & 3.70 & 1.13 & 0.80 & 0.81 & 1.21 & 1.82 & 1.65 & 0.69 & 0.17 \\
\hline & MEWMA_3 & $A A R L$ & 19.58 & 5.16 & 3.25 & 2.41 & 2.03 & 1.76 & 1.41 & 1.16 & 1.00 & 1.00 \\
\hline & & $S D A R L$ & 1.81 & 0.26 & 0.16 & 0.05 & 0.05 & 0.04 & 0.02 & 0.02 & 0.00 & 0.00 \\
\hline & & CVARL & 9.24 & 5.04 & 4.92 & 2.07 & 2.46 & 2.27 & 1.42 & 1.72 & 0.00 & 0.00 \\
\hline & MEWMA $/ \chi^{2}$ & $A A R L$ & 15.34 & 4.75 & 2.35 & 1.92 & 1.39 & 1.24 & 1.15 & 1.00 & 1.00 & 1.00 \\
\hline & & $S D A R L$ & 1.36 & 0.22 & 0.06 & 0.03 & 0.03 & 0.03 & 0.02 & 0.00 & 0.00 & 0.00 \\
\hline & & $C V A R L$ & 8.87 & 4.62 & 2.55 & 1.56 & 2.16 & 2.42 & 1.74 & 0.00 & 0.00 & 0.00 \\
\hline$\infty$ (Nooros- & MEWMA & $A R L$ & 14.80 & 4.90 & 3.00 & 2.20 & 1.90 & 1.60 & 1.30 & 1.00 & 1.00 & 1.00 \\
\hline (0) & MEWMA_3 & $A R L$ & 16.10 & 5.10 & 3.00 & 2.20 & 1.90 & 1.60 & 1.30 & 1.10 & 1.00 & 1.00 \\
\hline & MEWMA $/ \chi^{2}$ & $A R L$ & 13.70 & 4.50 & 2.60 & 1.80 & 1.30 & 1.10 & 1.00 & 1.00 & 1.00 & 1.00 \\
\hline
\end{tabular}


Table 5 AARL, SDARL and $C V A R L$ comparison when $\beta_{11}$ has shifted to $\beta_{11}+\tau_{1} \sigma_{1}$

\begin{tabular}{|c|c|c|c|c|c|c|c|c|c|c|c|c|}
\hline \multirow[t]{2}{*}{$m$} & \multirow[t]{2}{*}{ Method } & \multirow[t]{2}{*}{ Metric } & \multicolumn{10}{|l|}{$\tau_{1}$} \\
\hline & & & 0.025 & 0.05 & 0.075 & 0.1 & 0.125 & 0.15 & 0.175 & 0.2 & 0.225 & 0.25 \\
\hline \multirow[t]{9}{*}{30} & \multirow[t]{3}{*}{ MEWMA } & $A A R L$ & 42.08 & 9.64 & 5.22 & 3.60 & 2.79 & 2.45 & 2.11 & 1.94 & 1.72 & 1.57 \\
\hline & & $S D A R L$ & 20.14 & 1.49 & 0.57 & 0.24 & 0.11 & 0.09 & 0.08 & 0.06 & 0.04 & 0.01 \\
\hline & & CVARL & 47.86 & 15.46 & 10.92 & 6.67 & 3.94 & 3.67 & 3.79 & 3.25 & 2.33 & 0.64 \\
\hline & \multirow[t]{3}{*}{ MEWMA_3 } & $A A R L$ & 69.12 & 17.43 & 7.76 & 6.17 & 4.67 & 2.93 & 2.31 & 2.08 & 1.93 & 1.74 \\
\hline & & $S D A R L$ & 23.87 & 1.73 & 0.84 & 0.45 & 0.21 & 0.10 & 0.09 & 0.07 & 0.04 & 0.02 \\
\hline & & CVARL & 34.53 & 9.93 & 10.82 & 7.29 & 4.50 & 3.41 & 3.90 & 3.37 & 2.07 & 1.15 \\
\hline & \multirow[t]{3}{*}{ MEWMA/ $\chi^{2}$} & $A A R L$ & 49.23 & 9.86 & 5.86 & 3.84 & 2.71 & 2.18 & 1.75 & 1.68 & 1.59 & 1.42 \\
\hline & & SDARL & 20.29 & 2.82 & 0.66 & 0.27 & 0.14 & 0.12 & 0.06 & 0.05 & 0.03 & 0.01 \\
\hline & & CVARL & 41.21 & 28.60 & 11.26 & 7.03 & 5.17 & 5.50 & 3.43 & 2.98 & 1.89 & 0.70 \\
\hline \multirow[t]{9}{*}{50} & \multirow[t]{3}{*}{ MEWMA } & $A A R L$ & 41.98 & 9.62 & 5.21 & 3.59 & 2.76 & 2.42 & 2.09 & 1.92 & 1.72 & 1.56 \\
\hline & & $S D A R L$ & 20.27 & 1.32 & 0.38 & 0.18 & 0.08 & 0.08 & 0.06 & 0.05 & 0.02 & 0.01 \\
\hline & & CVARL & 48.28 & 13.72 & 7.29 & 5.01 & 2.90 & 3.31 & 2.87 & 2.60 & 1.16 & 0.64 \\
\hline & \multirow[t]{3}{*}{ MEWMA_3 } & $A A R L$ & 67.15 & 17.36 & 7.64 & 5.91 & 4.41 & 2.87 & 2.29 & 1.97 & 1.89 & 1.73 \\
\hline & & $S D A R L$ & 21.68 & 1.49 & 0.52 & 0.23 & 0.15 & 0.09 & 0.08 & 0.07 & 0.03 & 0.02 \\
\hline & & CVARL & 32.29 & 8.58 & 6.81 & 3.89 & 3.40 & 3.14 & 3.49 & 3.55 & 1.59 & 1.33 \\
\hline & \multirow[t]{3}{*}{ MEWMA $/ \chi^{2}$} & $A A R L$ & 48.25 & 9.76 & 5.61 & 3.79 & 2.66 & 2.16 & 1.72 & 1.63 & 1.59 & 1.38 \\
\hline & & $S D A R L$ & 19.56 & 1.38 & 0.42 & 0.21 & 0.09 & 0.08 & 0.07 & 0.05 & 0.02 & 0.01 \\
\hline & & CVARL & 40.54 & 14.14 & 7.49 & 5.54 & 3.38 & 3.70 & 4.07 & 3.07 & 1.26 & 0.72 \\
\hline \multirow[t]{9}{*}{100} & \multirow[t]{3}{*}{ MEWMA } & $A A R L$ & 39.36 & 9.57 & 5.18 & 3.54 & 2.75 & 2.37 & 2.08 & 1.90 & 1.72 & 1.52 \\
\hline & & $S D A R L$ & 14.07 & 1.01 & 0.37 & 0.16 & 0.08 & 0.05 & 0.03 & 0.02 & 0.01 & 0.01 \\
\hline & & CVARL & 35.75 & 10.55 & 7.14 & 4.52 & 2.91 & 2.11 & 1.44 & 1.05 & 0.58 & 0.66 \\
\hline & \multirow[t]{3}{*}{ MEWMA_3 } & $A A R L$ & 59.45 & 17.11 & 7.62 & 5.82 & 4.38 & 2.86 & 2.27 & 1.97 & 1.88 & 1.61 \\
\hline & & $S D A R L$ & 20.52 & 1.42 & 0.48 & 0.19 & 0.12 & 0.08 & 0.07 & 0.04 & 0.03 & 0.02 \\
\hline & & CVARL & 34.51 & 8.30 & 6.30 & 3.26 & 2.74 & 2.80 & 3.08 & 2.03 & 1.60 & 1.24 \\
\hline & \multirow[t]{3}{*}{ MEWMA $/ \chi^{2}$} & $A A R L$ & 46.21 & 9.65 & 5.53 & 3.78 & 2.64 & 2.13 & 1.67 & 1.45 & 1.58 & 1.36 \\
\hline & & SDARL & 16.54 & 1.31 & 0.42 & 0.18 & 0.08 & 0.06 & 0.05 & 0.05 & 0.03 & 0.01 \\
\hline & & CVARL & 35.79 & 13.58 & 7.59 & 4.76 & 3.03 & 2.82 & 2.99 & 3.45 & 1.90 & 0.74 \\
\hline \multirow[t]{9}{*}{300} & \multirow[t]{3}{*}{ MEWMA } & $A A R L$ & 39.20 & 9.31 & 5.11 & 3.53 & 2.74 & 2.33 & 2.05 & 1.89 & 1.71 & 1.52 \\
\hline & & SDARL & 4.81 & 0.70 & 0.24 & 0.12 & 0.08 & 0.04 & 0.03 & 0.02 & 0.01 & 0.01 \\
\hline & & CVARL & 12.27 & 7.52 & 4.70 & 3.40 & 2.92 & 1.72 & 1.46 & 1.06 & 0.58 & 0.66 \\
\hline & MEWMA_3 & $A A R L$ & 54.12 & 15.24 & 7.65 & 5.80 & 4.36 & 2.84 & 2.23 & 1.97 & 1.88 & 1.59 \\
\hline & & SDARL & 5.73 & 1.08 & 0.35 & 0.15 & 0.09 & 0.09 & 0.08 & 0.05 & 0.02 & 0.01 \\
\hline & & CVARL & 10.59 & 7.09 & 4.58 & 2.59 & 2.06 & 3.17 & 3.59 & 2.53 & 1.06 & 0.63 \\
\hline & MEWMA $/ \chi^{2}$ & $A A R L$ & 43.01 & 9.62 & 5.44 & 3.72 & 2.64 & 2.12 & 1.59 & 1.43 & 1.57 & 1.35 \\
\hline & & SDARL & 4.62 & 0.84 & 0.26 & 0.18 & 0.09 & 0.08 & 0.06 & 0.06 & 0.04 & 0.01 \\
\hline & & CVARL & 10.74 & 8.73 & 4.78 & 4.84 & 3.41 & 3.77 & 3.77 & 4.20 & 2.55 & 0.74 \\
\hline 600 & MEWMA & $A A R L$ & 37.34 & 9.22 & 5.00 & 3.51 & 2.70 & 2.28 & 2.03 & 1.88 & 1.69 & 1.46 \\
\hline & & SDARL & 3.24 & 0.53 & 0.15 & 0.08 & 0.04 & 0.03 & 0.02 & 0.02 & 0.01 & 0.01 \\
\hline & & CVARL & 8.68 & 5.75 & 3.00 & 2.28 & 1.48 & 1.18 & 0.99 & 1.06 & 0.59 & 0.68 \\
\hline & MEWMA_3 & $A A R L$ & 48.23 & 13.14 & 6.85 & 4.11 & 3.22 & 2.60 & 2.16 & 1.92 & 1.86 & 1.49 \\
\hline & & SDARL & 4.12 & 1.03 & 0.35 & 0.12 & 0.09 & 0.08 & 0.08 & 0.04 & 0.02 & 0.01 \\
\hline & & CVARL & 8.54 & 7.84 & 5.04 & 2.92 & 2.80 & 3.08 & 3.70 & 2.08 & 1.08 & 0.67 \\
\hline & MEWMA $/ \chi^{2}$ & $A A R L$ & 39.12 & 9.46 & 5.31 & 3.53 & 2.56 & 1.86 & 1.45 & 1.26 & 1.16 & 1.23 \\
\hline & & $S D A R L$ & 3.89 & 0.73 & 0.21 & 0.16 & 0.09 & 0.07 & 0.06 & 0.03 & 0.01 & 0.01 \\
\hline & & CVARL & 9.94 & 7.72 & 3.95 & 4.53 & 3.52 & 3.76 & 4.14 & 2.38 & 0.86 & 0.81 \\
\hline$\infty$ (Noorossana & MEWMA & $A R L$ & 30.20 & 8.60 & 4.80 & 3.30 & 2.60 & 2.20 & 2.00 & 1.80 & 1.60 & 1.40 \\
\hline et al. 2010) & MEWMA_3 & $A R L$ & 40.70 & 10.20 & 5.30 & 3.70 & 2.80 & 2.30 & 2.10 & 1.90 & 1.70 & 1.40 \\
\hline & MEWMA $/ \chi^{2}$ & $A R L$ & 34.40 & 9.10 & 4.90 & 3.30 & 2.40 & 1.80 & 1.40 & 1.20 & 1.10 & 1.00 \\
\hline
\end{tabular}


metric and needs fewer Phase I samples to achieve $90 \%$ of the desired in-control $A R L=200$. Although it is confidently inferred that the MEWMA_3 method performs better than the other competing methods in terms of the SDARL metric when $\theta=0.2$, the results are not the same for other values of $\theta$. We recommended using the CVARL metric as the basis of the comparison in order to simultaneously consider the AARL and SDARL. The results showed that the MEWMA_3 method performs uniformly better than other approaches in terms of the CVARL, although the MEWMA $/ \chi^{2}$ method performs better than the MEWMA_3 method for small values of $m$. The performance of the MEWMA_3 method improves by increasing $m$. After obtaining simulated corrected limits in order to reflect the variability added to the process by parameter estimation, we investigated the out-ofcontrol performance of the control chart schemes in terms of all the metrics, considering different out-of-control scenarios. The simulation results showed that the MEWMA and MEWMA $/ \chi^{2}$ charts have similar out-of-control performance in most cases. However, it can be declared that the MEWMA method generally performs better than other competing methods in terms of the AARL, SDARL and CVARL for all out-of-control scenarios.

Future research in this area could involve investigation of the parameter estimation effect on the in-control and outof-control performance of different monitoring approaches for other types of profiles, such as nonlinear or polynomial profiles. Also, new metrics could be used in order to better measure the effect of parameter estimation, which is very useful in deciding on superior methods.

Acknowledgments The authors thank to the anonymous referee for detailed and thoughtful recommendations which led to significant improvement in the paper. This research is partially supported by a grant from Iran National Science Foundation (INSF).

Open Access This article is distributed under the terms of the Creative Commons Attribution 4.0 International License (http://creativeco mmons.org/licenses/by/4.0/), which permits unrestricted use, distribution, and reproduction in any medium, provided you give appropriate credit to the original author(s) and the source, provide a link to the Creative Commons license, and indicate if changes were made.

\section{References}

Adibi A, Montgomery DC, Borror CM (2014) A $P$-value approach for phase II monitoring of multivariate profiles. Int J Qual Eng Technol 4(2):133-143

Aly AA, Mahmoud MA, Woodall WH (2015) A comparison of the performance of phase II simple linear profile control charts when parameters are estimated. Commun Stat Simul Comput 44(6): 1432-1440

Aly AA, Mahmoud MA, Hamed R (2016) The performance of the multivariate adaptive exponentially weighted moving average control chart with estimated parameters. Qual Reliab Eng Int 32(3):957-967

Amiri A, Eyvazian M, Zou C, Noorossana R (2012) A parameters reduction method for monitoring multiple linear regression profiles. Int J Adv Manuf Technol 58(5-8):621-629

Ayoubi M, Kazemzadeh RB, Noorossana R (2014) Estimating multivariate linear profiles change point with a monotonic change in the mean of response variables. Int $\mathbf{J}$ Adv Manuf Technol 75(9-12):1537-1556

Burroughs TE, Rigdon SE, Champ CW (1993) An analysis of Shewhart charts with runs rules when no standards are given. In: Proceedings of the quality and productivity section of the American statistical association, pp 8-12

Castagliola P, Maravelakis PE, Figueiredo FO (2016) The EWMA median chart with estimated parameters. IIE Trans 48(1):66-74

Chakraborti S (2000) Run length, average run length and false alarm rate of Shewhart X-bar chart: exact derivations by conditioning. Commun Stat Simul Comput 29(1):61-81

Champ CW, Jones-Farmer LA, Rigdon SE (2005) Properties of the $\mathrm{T}^{2}$ control chart when parameters are estimated. Technometrics 47(4):437-445

Chen G (1997) The mean and standard deviation of the run length distribution of $X$ charts when control limits are estimated. Stat Sin 7(3):789-798

Crowder S, Hamilton M (1992) An EWMA for monitoring a process standard deviation. J Qual Technol 24(1):12-21

Ding Y, Zeng L, Zhou S (2006) Phase I analysis for monitoring nonlinear profiles in manufacturing processes. J Qual Technol 38(3): 199-216

Gupta S, Montgomery DC, Woodall WH (2006) Performance evaluation of two methods for online monitoring of linear calibration profiles. Int J Prod Res 44(10):1927-1942

Jensen WA, Jones-Farmer LA, Champ CW, Woodall WH (2006) Effects of parameter estimation on control chart properties: a literature review. J Qual Technol 38(4):349-364

Jensen WA, Birch JB, Woodall WH (2008) Monitoring correlation within linear profiles using mixed models. J Qual Technol 40(2): 167-183

Jeong MK, Lu JC, Wang N (2006) Wavelet-based SPC procedure for complicated functional data. Int J Prod Res 44(4):729-744

Jones LA (2002) The statistical design of EWMA control charts with estimated parameters. J Qual Technol 34(3):277-288

Jones LA, Champ CW, Rigdon SE (2001) The performance of exponentially weighted moving average charts with estimated parameters. Technometrics 43(2):156-167

Jones LA, Champ CW, Rigdon SE (2004) The run length distribution of the CUSUM with estimated parameters. J Qual Technol 36(1):95-108

Kalaei M, Soleimani P, Niaki STA, Atashgar K (2018) Phase-I monitoring of standard deviations in multistage linear profiles. J Ind Eng Int 14(1):133-142

Kang L, Albin SL (2000) On-line monitoring when the process yields a linear profile. J Qual Technol 32(4):418-426

Kazemzadeh RB, Noorossana R, Amiri A (2008) Phase I monitoring of polynomial profiles. Commun Stat Theory Methods 37(10):1671-1686

Kazemzadeh RB, Noorossana R, Amiri A (2009) Monitoring polynomial profiles in quality control applications. Int J Adv Manuf Technol 42(7):703-712

Khedmati M, Niaki STA (2015) Identifying the time of a step change in AR (1) auto-correlated simple linear profiles. J Ind Eng Int 11(4):473-484

Khedmati M, Niaki STA (2016) Monitoring simple linear profiles in multistage processes by a MaxEWMA control chart. Comput Ind Eng 98:125-143 
Khoo MBC (2005) A control chart based on sample median for the detection of a permanent shift in the process mean. Qual Eng 17(2):243-257

Kim K, Mahmoud MA, Woodall WH (2003) On the monitoring of linear profiles. J Qual Technol 35(3):317-328

Lowry CA, Woodall WH, Champ CW, Rigdon SE (1992) A multivariate exponentially weighted moving average control chart. Technometrics 34(1):46-53

Mahmoud MA (2008) Phase I analysis of multiple linear regression profiles. Commun Stat Simul Comput 37(10):2106-2130

Mahmoud MA (2012) The performance of phase II simple linear profile approaches when parameters are estimated. Commun Stat Simul Comput 41(10):1816-1833

Mahmoud MA, Maravelakis PE (2010) The performance of the MEWMA control chart when parameters are estimated. Commun Stat Simul Comput 39(9):1803-1817

Mahmoud MA, Woodall WH (2004) Phase I analysis of linear profiles with calibration applications. Technometrics 46(4):380-391

Mahmoud MA, Parker PA, Woodall WH, Hawkins DM (2007) A change point method for linear profile data. Qual Reliab Eng Int 23(2):247-268

Mahmoud MA, Morgan JP, Woodall WH (2010) The monitoring of simple linear regression profiles with two observations per sample. J Appl Stat 37(8):1249-1263

Mestek O, Pavlík J, Suchánek M (1994) Multivariate control charts: control charts for calibration curves. Fresenius' J Anal Chem 350(6):344-351

Moguerza JM, Muñoz A, Psarakis S (2007) Monitoring nonlinear profiles using support vector machines. In: Iberoamerican congress on pattern recognition

Narvand A, Soleimani P, Raissi S (2013) Phase II monitoring of autocorrelated linear profiles using linear mixed model. J Ind Eng Int 9(1):1-12

Noorossana R, Amiri A, Vaghefi A, Roghanian E (2004) Monitoring quality characteristics using linear profile. In: Proceeding of the 3rd international industrial engineering conference, Tehran, Iran

Noorossana R, Amiri A, Soleimani P (2008) On the monitoring of autocorrelated linear profiles. Commun Stat Theory Methods 37(3):425-442

Noorossana R, Eyvazian M, Vaghefi A (2010) Phase II monitoring of multivariate simple linear profiles. Comput Ind Eng 58(4):563-570

Noorossana R, Saghaei A, Amiri A (2011) Statistical analysis of profile monitoring, vol 865. Wiley, New York

Parker PA, Finley TD (2007) Advancements in aircraft model force and attitude instrumentation by integrating statistical methods. J Aircr 44(2):436-443

Psarakis S, Vyniou AK, Castagliola P (2014) Some recent developments on the effects of parameter estimation on control charts. Qual Reliab Eng Int 30(8):1113-1129
Quesenberry CP (1993) The effect of sample size on estimated limits for X control charts. J Qual Technol 25(4):237-247

Saleh NA, Mahmoud MA, Jones-Farmer LA, Zwetsloot I, Woodall WH (2015) Another look at the EWMA control chart with estimated parameters. J Qual Technol 47(4):363

Shishebori D, Zeinal Hamadani A (2009) The effect of gauge measurement capability on MC p and its statistical properties. Int J Qual Reliab Manag 26(6):564-582

Shishebori D, Akhgari MJ, Noorossana R, Khaleghi GH (2015) An efficient integrated approach to reduce scraps of industrial manufacturing processes: a case study from gauge measurement tool production firm. Int J Adv Manuf Technol 76(5-8):831-855

Shu L, Tsung F, Kwok-Leung T (2004) Run-length performance of regression control charts with estimated parameters. J Qual Technol 36(3):280-292

Stover FS, Brill RV (1998) Statistical quality control applied to ion chromatography calibrations. J Chromatogr 804(1-2):37-43

Vaghefi A, Tajbakhsh SD, Noorossana R (2009) Phase II monitoring of nonlinear profiles. Commun Stat Theory Methods 38(11):1834-1851

Wang K, Tsung F (2005) Using profile monitoring techniques for a data-rich environment with huge sample size. Qual Reliab Eng Int 21(7):677-688

Williams JD, Woodall WH, Birch JB (2007) Statistical monitoring of nonlinear product and process quality profiles. Qual Reliab Eng Int 23(8):925-941

Woodall WH (2007) Current research on profile monitoring. Production 17(3):420-425

Woodall WH, Montgomery DC (2014) Some current directions in the theory and application of statistical process monitoring. J Qual Technol 46(1):78-94

Woodall WH, Spitzner DJ, Montgomery DC, Gupta S (2004) Using control charts to monitor process and product quality profiles. J Qual Technol 36(3):309-320

Zhang L, Chen G (2002) A note on EWMA charts for monitoring mean changes in normal processes. Commun Stat Theory Methods 31(4):649-661

Zhang M, Megahed FM, Woodall WH (2014) Exponential CUSUM charts with estimated control limits. Qual Reliab Eng Int 30(2):275-286

Zou C, Tsung F, Wang Z (2007) Monitoring general linear profiles using multivariate exponentially weighted moving average schemes. Technometrics 49(4):395-408

Zou C, Ning X, Tsung F (2012) LASSO-based multivariate linear profile monitoring. Ann Oper Res 192(1):3-19

Zwetsloot IM, Woodall WH (2017) A head-to-head comparative study of the conditional performance of control charts based on estimated parameters. Qual Eng 29(2):244-253 